\title{
Análise bibliométrica sobre abordagens da gestão universitária em publicações na plataforma Web of Science entre 1945 a 2020
}

Bibliometric analysis on university management approaches in publications on the Web of Science platform between 1945 and 2020

Análisis bibliométrico sobre enfoques de gestión universitaria en publicaciones en la plataforma Web of Science entre 1945 y 2020

\author{
Allan Gustavo Freire da Silva \\ ORCID: https://orcid.org/0000-0003-1550-8061 \\ Universidade Federal de Campina Grande, Brasil \\ E-mail: allangfs@hotmail.com \\ Laís Karla da Silva Barreto \\ ORCID: https://orcid.org/0000-0002-5267-5355 \\ Universidade Potiguar, Brasil \\ E-mail: laisbarreto@gmail.com \\ Marcelo Victor Alves Bila Queiroz \\ ORCID: https://orcid.org/0000-0001-6182-8600 \\ Universidade Potiguar, Brasil \\ E-mail: marcelo.queiroz@unp.br \\ Rômulo Andrade de Souza Neto \\ ORCID: https://orcid.org/0000-0003-1810-939X \\ Universidade Potiguar, Brasil \\ E-mail: romulo.souza@unp.br \\ Emilia Suitberta de Oliveira Trigueiro \\ ORCID: https://orcid.org/0000-0002-7649-7688 \\ Centro Universitário Dr. Leão Sampaio, Brasil \\ E-mail: emiliasuitberta@leaosampaio.edu.br
}

\begin{abstract}
Resumo
Transformações sociais e econômicas somadas às medidas governamentais registradas em normas e decretos têm impulsionado discussões no âmbito público, visando maior eficiência nas ações, melhorias na prestação de serviços educacionais e fomento à inovação. Nesse sentido, a gestão universitária passa a ser o foco de modernizações e buscas por maior satisfação de usuários diante da prestação dos serviços públicos. Sobre essas questões, esse trabalho tem como objetivo analisar os direcionamentos de pesquisas voltadas à gestão universitária, registradas como artigos científicos na plataforma Web of Science, entre o período de 1945 a 2020. Metodologicamente, trata-se de pesquisa com abordagem quantitativa, fundamentada a partir de análise bibliométrica em plataforma Web of Science, abrangendo o período de consulta de 1945 a 2020. Como resultados, registram-se índices de publicação, especificidades de pesquisas mais relevantes na área da gestão universitária, autores mais citados e ocorrências de palavras. Conclui-se que diante dos estudos mais relevantes, disponíveis na Web of Science, não há abordagens integradas que envolvam questões como eficiência, satisfação de usuários e inovação na gestão educacional superior, especialmente, considerando o setor público brasileiro.
\end{abstract}

Palavras-chave: Gestão universitária; Eficiência; Satisfação; Setor público.

\begin{abstract}
Social and economic transformations added to governmental measures registered in norms and decrees have stimulated discussions in the public sphere, aiming at greater efficiency in actions, improvements in the provision of educational services and fostering innovation. In this sense, university management becomes the focus of modernization and the search for greater user satisfaction with the provision of public services. On these issues, this work aims to analyze the directions of research aimed at university management, registered as scientific articles on the Web of Science platform, between the period from 1945 to 2020. Methodologically, it is a research with a quantitative approach, based on the from bibliometric analysis on the Web of Science platform, covering the period of consultation from 1945 to 2020. As a result, publication rates, specificities of more relevant research in the area of university management, most cited authors and occurrences of words are recorded. It is concluded that, given the most relevant
\end{abstract}


studies available on the Web of Science, there are no integrated approaches that involve issues such as efficiency, user satisfaction and innovation in higher education management, especially considering the Brazilian public sector.

Keywords: University management; Efficiency; Satisfaction; Public Sector.

\begin{abstract}
Resumen
Las transformaciones sociales y económicas sumadas a las medidas gubernamentales registradas en normas y decretos han estimulado discusiones en la esfera pública, buscando mayor eficiencia en las acciones, mejoras en la prestación de los servicios educativos y fomento de la innovación. En este sentido, la gestión universitaria se convierte en el foco de la modernización y de la búsqueda de una mayor satisfacción de los usuarios con la prestación de los servicios públicos. Sobre estos temas, este trabajo tiene como objetivo analizar los rumbos de la investigación dirigida a la gestión universitaria, registrados como artículos científicos en la plataforma Web of Science, entre el período de 1945 a 2020. Metodológicamente, se trata de una investigación con enfoque cuantitativo, basada en a partir del análisis bibliométrico en la plataforma Web of Science, abarcando el período de consulta desde 1945 hasta 2020. Como resultado se muestran tasas de publicación, especificidades de las investigaciones más relevantes en el área de la gestión universitaria, autores más citados y ocurrencias de palabras. grabado. Se concluye que, dados los estudios más relevantes disponibles en la Web of Science, no existen enfoques integrados que involucren cuestiones como la eficiencia, la satisfacción del usuario y la innovación en la gestión de la educación superior, especialmente considerando el sector público brasileño.
\end{abstract}

Palabras clave: Gestión universitaria; Eficiencia; Satisfacción; Sector público.

\title{
1. Introdução
}

Novas demandas sociais, econômicas e políticas estão entre os fatores que incitam mudanças nas organizações públicas e privadas. A competitividade do mercado, a racionalização de recursos, o surgimento de novas tecnologias, além da necessidade de melhores resultados e ações, inserem as instituições em diversificados e desafiadores cenários (Soares et al., 2019), de buscas por modernização administrativa e processual, alcançando múltiplos setores e ambientes org anizacionais.

As normas federais, voltadas aos serviços públicos, instituídas na Lei no 9.394/1996, contemplam, no Art. $3^{\circ}$, dentre suas características o princípio "IX - garantia de padrão de qualidade". Tal expressão, considerando o ensino público federal superior, ratifica o compromisso governamental de que seja perseguido pelos serviços públicos da educação critérios que fomentem a eficácia, a padronização no fornecimento do ensino, atentando-se ainda, às características que evidenciem a transparência dos seus atos, conforme estabelecido no $§ 1^{\circ}$ do Art. 47 da supracitada Lei, referendada, pela Lei $n^{\circ}$ 13.168/2015.

Critérios para criação de setores, atividades inerentes a cada ambiente, rito processual, demandas internas e externas às questões educacionais, otimização de recursos públicos, o tempo de resposta institucional e a qualidade da comunicação interinstitucional se inserem na necessidade de composição de um arcabouço de transparência e de eficiência organizacional, princípios basilares ao fornecimento dos serviços públicos, tonificados pela Lei $\mathrm{n}^{\circ}$ 13.460/2017, Art $5^{\circ}$, XIII, ao evidenciar, como direito dos usuários e dever dos agentes públicos a "aplicação de soluções tecnológicas que visem a simplificar processos e procedimentos de atendimento ao usuário e a propiciar melhores condições para o compartilhamento das informações".

Como problema central de pesquisa, tem-se o seguinte questionamento: quais são as abordagens realizadas pela literatura no tocante às discussões sobre gestão universitária e seus fatores de eficiência, inovação e satisfação de usuários?

Tem-se como objetivo geral analisar os direcionamentos de pesquisas voltadas à gestão universitária, registradas como artigos científicos na plataforma Web of Science, entre o período de 1945 a 2020. Tendo como objetivos específicos: Relacionar abordagens dos artigos considerando perspectivas de satisfação de usuários e eficiência no ensino público e inovação; Descrever possíveis enfoques de pesquisa na área da Gestão Universitária a partir da incidência de expressões registradas em cada trabalho; Promover exame e sistematização de informações, de modo a apresentar diagnósticos e promover fortalecimento de novos estudos que impulsionem pesquisas na área. 
Como justificativa, repara-se que o aprofundamento destas análises diversifica e amplia a capacidade resolutiva de problemas sociais, ao reconhecer o ambiente universitário como relevante parte do processo de impulsionamento e transformações sociais, salientando seu vínculo social e busca por soluções (Li et al., 2018).

Destarte, a relevância dos estudos - ora em comento, voltados à temática da gestão universitária - apresenta ampla perspectiva, ao frisar abordagens que abarcam tanto o processo decisório de gestores, seus aspectos de motivação e satisfação no meio acadêmico, e também, como conselhos influenciam no direcionamento de recursos e suas implicações em atividades de ensino e pesquisa.

Os resultados apontam que, não obstante, mesmo havendo múltiplas abordagens sobre o meio acadêmico universitário, as mais relevantes pesquisas evidenciadas na plataforma de pesquisa Web of Science, não contemplam visões de estudos que englobem análises de modernização administrativa, de otimização de processos e sua possível influência na estrutura organizacional, no rendimento acadêmico e na satisfação de usuários do serviço público educacional superior, sobretudo, quando posta esta análise perante contexto de instituição pública federal, proveniente de política pública de expansão universitária brasileira e democratização do ensino superior.

O trabalho encontra-se estruturado com análises sobre a gestão universitária, abordagens sobre tecnologias, satisfação de usuários, eficiência e inovação no contexto das universidades; apresenta discussão sobre quantitativos de trabalhos, relevância e adequações à temática da gestão universitária; e considerações sobre pontos tidos como essenciais para o desenvolvimento da temática, alcance de objetivos do artigo e resposta ao problema de pesquisa.

\section{Metodologia}

Trata-se de pesquisa que se apresenta com abordagem quantitativa, posto que busca-se interpretar e registrar aspectos subjetivos, inerentes às dinâmicas organizacionais no cenário acadêmico, público e superior. Assim, processos e focos de avaliação exsurgem com base na interpretação e visão do pesquisador fundamentadas na teoria, porém, sem o uso de estatística como prioridade, para posterior a análise e resposta a determinado problema de pesquisa (Prodanov \& Freitas, 2013).

Dada a relevância da temática referente à otimização de processos e eficiência no contexto acadêmico superior, ao mensurar o quantitativo de estudos que abordam essa discussão, compreendendo a base de pesquisa e seus bancos de dados: Web of Science, tendo em vista que, conforme Csomós (2020), considerando o contexto internacional, plataforma em estudo é uma das mais utilizadas para a realização de análises bibliométricas, dado suas vantagens de instrumentos analíticos, filtragens, e relevante cobertura e portfólio de artigos.

A partir de análises na plataforma Web of Science observa-se a incidência de termos e específicos agrupamentos de palavras, formando clusters com os temas mais discutidos no meio acadêmico e publicados nos mais variados periódicos do mundo. Atividade esta, de mensuração e quantificação de estudos, fortalecida mediante a afirmação de Oliveira (2018), ao comentar que a medição da atividade científica é fundamental em todos os campos do conhecimento.

Para o desdobramento desse estudo, por meio da fragmentação de seus conteúdos, baseou-se nos escritos de Pereira et al. (2019), para traçar os mecanismos de abordagem e realizar sistemática revisão dos estudos publicados. Trata-se, pois, de análise fundamentada em levantamento sistemático da literatura direcionada ao tema da gestão universitária, considerando para isso, úberes autores - mais citados dentre os demais pesquisadores no período compreendido de análise - em suas respectivas áreas de estudo.

Para tais análises e correlações morfológicas, utilizou-se de software VOSviewer, para traçar relações de intensidade de estudos, destaques de palavras, enfoques e direcionamentos de análises sobre termos e semânticas, áreas e conteúdos de avaliação específicos, voltados ao meio acadêmico universitário. 
Consoante ao desenvolvimento dessa análise, realizada mediante consultas à plataforma Web of Science em 28 de julho de 2020, priorizou-se trabalhos com tipo de documento classificado como artigos publicados e considerou-se toda a faixa temporal disponível no banco de dados da Web of Science, o qual versa de 1945 a 2020. Atentou-se como definição de palavra-chave para a realização das buscas, o termo "university* management*" (gestão universitária), de modo a abarcar todas as pesquisas que correlacionassem o meio universitário como objeto central de estudo.

Por se tratar de estudo que se debruça sobre as análises que envolvem o âmbito universitário, diante de diversos contextos de pessoas, tecnologia e estrutura organizacional específica, considerou-se o termo "university management" como expressão norteadora e generalizada, para a realização da pesquisa nas bases de pesquisa Web of Science, onde, tendo em vista, a variedade de estudos na área, trata-se de termo genérico, o qual visou abarcar, em seus processos de avaliação, o maior número de discussões e contextos de artigos que envolvem a temática da gestão das universidades e suas nuances.

Dessa pesquisa na plataforma Web of Science, obteve-se um total de 492 publicações, categorizados em diversas áreas do conhecimento, a citar as principais para esse estudo e seus quantitativos: Educação Pesquisa Educacional, com 179 trabalhos; Gestão, com 87 artigos; Negócios, apresentando 44 trabalhos; Ciência da Informação/Ciência da Biblioteca, possuindo 37 artigos relacionados; Economia, com 28 artigos; e Ciências Sociais Interdisciplinares com 27 artigos.

\section{Resultados e Discussão}

Nesta seção, expõem-se dados referentes a quantitativos de publicações e especificações das abordagens de trabalhos relacionados à gestão universitária. É também estabelecido, análises sobre níveis de citação e ocorrências de palavras - ante avaliação temporal - em trabalhos pesquisados na plataforma Web of Science.

\subsection{Análise de metadados e geração de redes}

A supracitada expressão, cerne de análise de metadados, é utilizada com maior direcionamento, considerando o fito discursivo dessa pesquisa, em trabalhos relativos à lide acadêmica e suas particularidades, vasta e/ou estreitamente, por autores como Lacy e Sheehan (1997); Knott e Payne (2004); O'kane et al. (2015).

Conforme Gráfico 1, salienta-se que as publicações cujo tema central seja a gestão universitária, se intensificam a partir de 2015, sobretudo, em 2017 e 2019. Ressalta-se que mesmo compreendendo o período de análise de publicações mundiais na base da Web of Science, de 1945 a 2020, sobre o termo pesquisado, a contagem com a incidência de publicações, se inicia em 1996, demonstrando assim, o despertar científico sobre a gestão organizacional acadêmica, a qual assume protagonismo à medida que se constitui com a possibilidade de ser fator de diferenciação no tocante à maximização de eficiência, satisfação, excelência e foco em resultados. 
Gráfico 1. Publicações por ano - Web of Science.

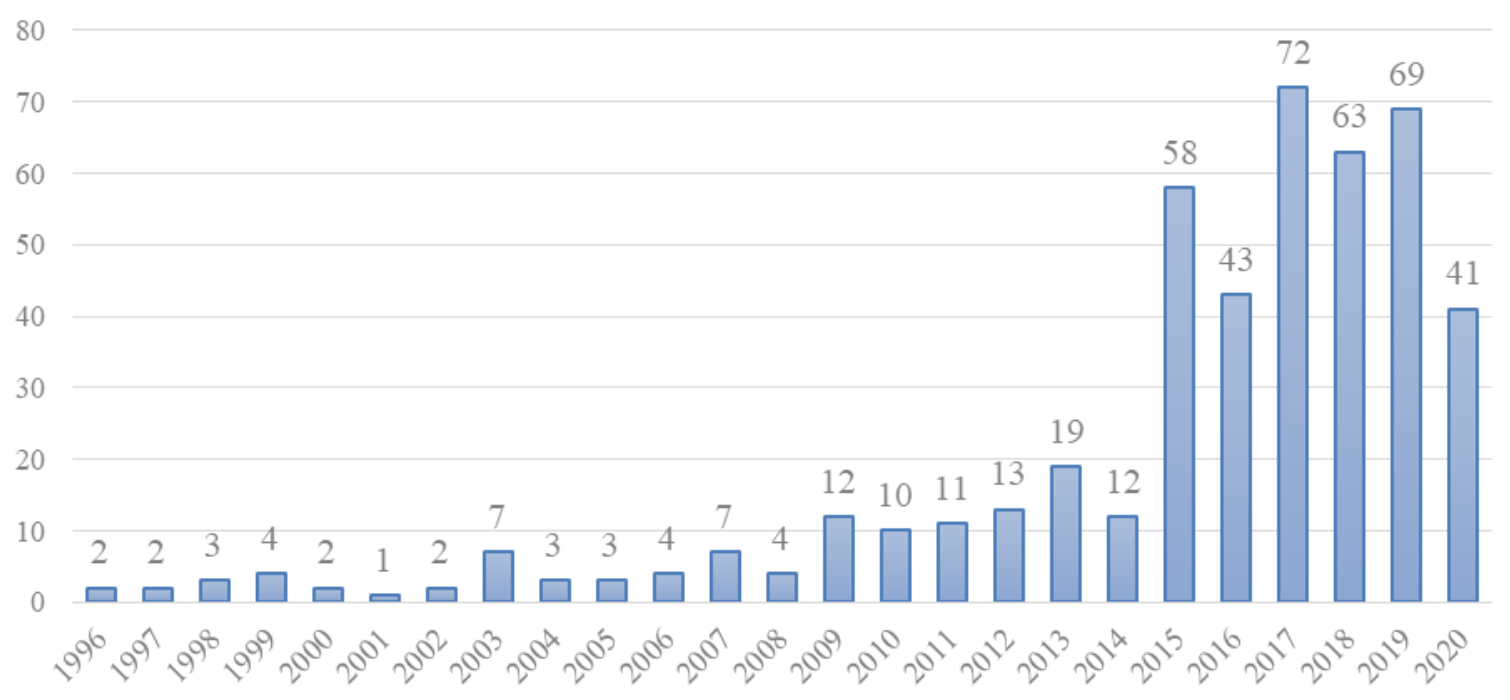

Fonte: Elaborado pelos autores (2020).

Neste cenário da educação superior, compreende-se que ações que focalizem na ampliação da eficiência e eficácia dos serviços, conforme ensejadas, sobretudo, por medidas governamentais motivadas pelo Decreto $\mathrm{n}^{\circ}$ 5.378/2005; Lei 13.460 de 2017; e Decreto 9.094/2017, são mudanças que convergem para perspectivas transformativas, impulsionadas pela globalização e contexto de promoção de educação, por meio de instituições públicas e privadas. Estes são entendimentos e percepções que podem ter corroborado para o aumento de estudos nessa temática, conforme enfatizado no Gráfico 1.

Referente às especificidades de cada trabalho, arrolado dentre os autores da Web of Science, registra-se no Quadro 1, a descrição de cada obra evidenciada dentre as mais relevantes sobre o termo "gestão universitária", a partir do entendimento de convergência discursiva, direta e/ou indireta, direcionada aos objetivos da pesquisa em tela e problema em questão considerando para isso, as formas de abordagem de análises sobre a universidade, seus formatos de gestão e atores internos ao meio acadêmico, registrados em cada artigo avaliado proveniente de autores da Web of Science - ante o amplo contexto da temática.

Sobre as pesquisas e descrições dos objetos de análise em cada estudo elencado, considerando o problema dessa pesquisa e os desígnios deste trabalho, destacam-se os estudos dos autores: O'Kane et al. (2015), ao enfatizar as universidades como escritórios de tecnologia e fomentadoras de legitimidade e de perspectivas entre acadêmicos; Lacy e Sheehan (1997), ao relacionar aspectos de satisfação acadêmica com o clima organizacional e fatores de integração e engajamento; Knott e Payne (2004) os quais registram em seus escritos como a estrutura de conselhos no âmbito estadual universitário distribuem recursos e como gestores lidam com ações de ensino e pesquisa.

Evidenciados pelos aludidos autores, elementos como tecnologia e perspectivas entre acadêmicos; satisfação acadêmica, engajamento e clima organizacional; e ações organizacionais de distribuição de recursos, podem ser critérios que fomentem substanciais impactos e alterações em resultados e ações de ensino e pesquisa no meio universitário.

Estas são, portanto, pesquisas que visam, de modo e prismas metodológicos diferenciados, analisar aspectos de ensino, pesquisa ou fatores administrativos que impactam a satisfação de usuários dos serviços públicos e possuem a capacidade de alterar resultados organizacionais. 
Quadro 1. Especificidades de pesquisas Web of Science - Por autores mais citados.

\begin{tabular}{|c|c|c|c|}
\hline Autores & Revista & Título & Descrição \\
\hline $\begin{array}{l}\text { Kaplan, Andreas } \\
\text { M.; Haenlein, } \\
\text { Michael }\end{array}$ & $\begin{array}{l}\text { Business } \\
\text { Horizons }\end{array}$ & $\begin{array}{l}\text { Higher education and the } \\
\text { digital revolution: About } \\
\text { MOOCs, SPOCs, social } \\
\text { media, and the Cookie } \\
\text { Monster }\end{array}$ & $\begin{array}{l}\text { Análise sobre o ambiente online de ensino à distância. Sua } \\
\text { evolução e conceitos e seus benefícios às instituições por } \\
\text { meio do instrumento de ensino remoto. }\end{array}$ \\
\hline $\begin{array}{l}\text { Pucciarelli, } \\
\text { Francesca; } \\
\text { Kaplan, Andreas }\end{array}$ & $\begin{array}{l}\text { Business } \\
\text { Horizons }\end{array}$ & $\begin{array}{l}\text { Competition and strategy } \\
\text { in higher education: } \\
\text { Managing complexity } \\
\text { and uncertainty }\end{array}$ & $\begin{array}{c}\text { Inseridas em contexto de competição de relações público e } \\
\text { privadas para o fornecimento da educação superior, } \\
\text { aborda-se tendências que influenciam essas organizações, } \\
\text { por meio de análise SWOT. Surge a necessidade de } \\
\text { estratégias e orientações para gestores e formulação de } \\
\text { políticas. }\end{array}$ \\
\hline $\begin{array}{l}\text { O'Kane, Concir; } \\
\text { Mangematin, } \\
\text { Vincent; } \\
\text { Geoghegan, Will; } \\
\text { Fitzgerald, Ciara }\end{array}$ & Research Policy & $\begin{array}{l}\text { University technology } \\
\text { transfer offices: The } \\
\text { search for identity to } \\
\text { build legitimacy }\end{array}$ & $\begin{array}{c}\text { Discorre sobre a importância dos Escritórios de } \\
\text { Transferência de Tecnologia (ETT) para as universidades. } \\
\text { Abordam o ETT como ferramenta promotora de } \\
\text { legitimidade, alterando visões de gestões e de acadêmicos } \\
\text { universitários. }\end{array}$ \\
\hline $\begin{array}{l}\text { Wilkins, Stephen; } \\
\text { Huisman, Jeroen }\end{array}$ & Higher Education & $\begin{array}{l}\text { The international branch } \\
\text { campus as transnational } \\
\text { strategy in higher } \\
\text { education }\end{array}$ & $\begin{array}{l}\text { Reflexões sobre determinações das universidades para } \\
\text { envolvimento e criação de filiais internacionais. Fatores } \\
\text { como níveis de risco, distância institucional, status e } \\
\text { legitimidade, dinamizam esse processo. }\end{array}$ \\
\hline $\begin{array}{l}\text { Thürer, Matthias; } \\
\text { Stevenson', Mark; } \\
\text { Silva, Cristovao; } \\
\text { Land, Martin J.; } \\
\text { Fredendall, } \\
\text { Lawrence D. } \\
\end{array}$ & $\begin{array}{l}\text { Production and } \\
\text { Operations } \\
\text { Management }\end{array}$ & $\begin{array}{l}\text { Workload Control and } \\
\text { Order Release: A Lean } \\
\text { Solution for Make-to- } \\
\text { Order Companies }\end{array}$ & $\begin{array}{l}\text { Avalia o rendimento de tempos de trabalho a partir de } \\
\text { análises sobre pedidos e despachos, considerando para } \\
\text { isso, atuações em pequenas e médias organizações. }\end{array}$ \\
\hline $\begin{array}{l}\text { Tian, Jing; } \\
\text { Nakamori, } \\
\text { Yoshiteru; } \\
\text { Wierzbicki, } \\
\text { Andrzej P. }\end{array}$ & $\begin{array}{l}\text { Journal of } \\
\text { Knowledge } \\
\text { Management }\end{array}$ & $\begin{array}{l}\text { Knowledge management } \\
\text { and knowledge creation } \\
\text { in academia: a study } \\
\text { based on surveys in a } \\
\text { Japanese research } \\
\text { university } \\
\end{array}$ & $\begin{array}{l}\text { Visa como utilizar o conhecimento de gestão para } \\
\text { aprimorar a atuação das universidades. Traz à discussão de } \\
\text { que abordagens de gestão do conhecimento também são } \\
\text { aplicáveis à academia. }\end{array}$ \\
\hline $\begin{array}{l}\text { Huisman, J; } \\
\text { Currie, J }\end{array}$ & Higher Education & $\begin{array}{l}\text { Accountability in higher } \\
\text { education: Bridge over } \\
\text { troubled water? }\end{array}$ & $\begin{array}{c}\text { Aborda a responsabilização de políticas de ensino superior } \\
\text { nos Estados Unidos e Europa. Discute-se a relação entre } \\
\text { autonomia, ensino superior e governos, e como a } \\
\text { responsabilidade e globalização estão presentes em } \\
\text { políticas educacionais. }\end{array}$ \\
\hline $\begin{array}{c}\text { Knott, JH; Payne, } \\
\text { AA }\end{array}$ & $\begin{array}{l}\text { Journal of Policy } \\
\text { Analysis And } \\
\text { Management }\end{array}$ & $\begin{array}{l}\text { The impact of state } \\
\text { governance structures on } \\
\text { management and } \\
\text { performance of public } \\
\text { organizations: A study of } \\
\text { higher education } \\
\text { institutions }\end{array}$ & $\begin{array}{l}\text { Visa analisar como a estrutura de conselhos estaduais de } \\
\text { ensino superior influencia no formato como gestores } \\
\text { distribuem recursos, buscam novas formas de receitas e } \\
\text { novos modelos de fomento ao ensino e pesquisa na } \\
\text { graduação. }\end{array}$ \\
\hline $\begin{array}{l}\text { Ylijoki, OH; } \\
\text { Mantyla, H }\end{array}$ & Time \& Society & $\begin{array}{l}\text { Conflicting time } \\
\text { perspectives in academic } \\
\text { work }\end{array}$ & $\begin{array}{c}\text { Discute recentes mudanças na gestão e financiamento da } \\
\text { universidade. O tempo situa-se como fundamental aspecto } \\
\text { da experiência humana e vida social. Relevância temporal } \\
\text { na compreensão e experiências dos indivíduos no ambiente } \\
\text { organizacional }\end{array}$ \\
\hline $\begin{array}{c}\text { Lacy, FJ; } \\
\text { Sheehan, BA }\end{array}$ & Higher Education & $\begin{array}{l}\text { Job satisfaction among } \\
\text { academic staff: An } \\
\text { international perspective }\end{array}$ & $\begin{array}{l}\text { Analisa aspectos de satisfação daqueles que integram a } \\
\text { academia e estuda seus níveis de clima acadêmico em oito } \\
\text { nações. Sobre níveis de satisfação no trabalho, inserem no } \\
\text { ambiente fatores referentes à moral, senso de comunidade } \\
\text { e relacionamento com colegas. }\end{array}$ \\
\hline
\end{tabular}

Fonte: Elaborado pelos autores (2020).

Dentre os autores seminais identificados, a partir de pesquisa realizada em base de dados Web of Science, e que discutem, em seus estudos, temáticas ligadas à gestão universitária, evidencia-se os autores mais citados, considerando obras publicadas entre os anos de 1997 a 2016 - sendo este período, a faixa temporal de obras publicadas e correlacionadas com 
citações, disponível para coleta de dados e avaliações sobre a supra temática na respectiva base de pesquisa, de acordo com a Tabela 1.

Trata-se, portanto, do conjunto de trabalhos publicados, relacionando à série história de publicação de artigos, dispondo de dados sobre o quantitativo de citações de autores arrolados na Tabela 1, de 2000 a 2020 - sendo este, o período máximo, referente aos autores mais citados, em artigos de destaque na temática da "gestão universitária", com os autores mais relevantes e suas respectivas publicações, a partir dos dados acessíveis em banco de informações da plataforma Web of Science.

Na Tabela 1, tem-se a listagem de autores em ordem decrescente de ano de publicação de seus trabalhos, destacando, o total de publicação. Ressalta-se o crescimento quantitativo de citações, entre 2000 a 2020, havendo maior incidência de citações recentes, os autores Kaplan Andreas e Michael Haenlein, ao possuírem no ano de 2019, 47 citações, referentes à pesquisa sobre a temática "gestão universitária”, publicadas em 2016, totalizando, para o período pesquisado, 108 citações destes autores, concentradas entre os anos de 2016 a 2020.

No entanto, citações melhor distribuídas, são registradas sobre os autores Ylijoki e Mantyla, atinente à publicação de 2003, os quais foram citados 114 vezes, entre 2004 a 2020, tal comportamento, realça a constância de atualização de publicação registrada em 2013, sendo também tidos assim, como autores relevantes e com continuada citação de sua obra.

A Tabela 1 discrimina os índices de citação de cada trabalho elencado na base Web of Science, o qual, ordenado conforme ano de publicação e autores, notabiliza as citações em seus respectivos anos, expressando dessa forma, a relevância das pesquisas, com suas variadas perspectivas sobre o tema da gestão universitária. $\mathrm{O}$ arrolamento temporal da análise assenta a importância de abordagem dos conteúdos, com registros de trabalhos, 1997 a 2016, dentre os mais citados na área. 
Tabela 1. Autores mais citados - Web of Science.

\begin{tabular}{|c|c|c|c|c|c|c|c|c|c|c|c|c|c|c|c|c|c|c|c|c|}
\hline Autores mais citados & $\begin{array}{c}\text { Ano da } \\
\text { publicação }\end{array}$ & 2000 & 2004 & 2005 & 2006 & 2007 & 2008 & 2009 & 2010 & 2011 & 2012 & 2013 & 2014 & 2015 & 2016 & 2017 & 2018 & 2019 & 2020 & Total \\
\hline $\begin{array}{l}\text { Kaplan, Andreas M.; } \\
\text { Haenlein, Michael }\end{array}$ & 2016 & 0 & 0 & 0 & 0 & 0 & 0 & 0 & 0 & 0 & 0 & 0 & 0 & 0 & 1 & 18 & 25 & 47 & 17 & 108 \\
\hline $\begin{array}{l}\text { Pucciarelli, Francesca; } \\
\text { Kaplan, Andreas }\end{array}$ & 2016 & 0 & 0 & 0 & 0 & 0 & 0 & 0 & 0 & 0 & 0 & 0 & 0 & 0 & 4 & 9 & 18 & 29 & 20 & 80 \\
\hline $\begin{array}{l}\text { O'Kane, Concir; } \\
\text { Mangematin, Vincent; } \\
\text { Geoghegan, Will; } \\
\text { Fitzgerald, Ciara }\end{array}$ & 2015 & 0 & 0 & 0 & 0 & 0 & 0 & 0 & 0 & 0 & 0 & 0 & 0 & 2 & 8 & 14 & 20 & 17 & 13 & 74 \\
\hline $\begin{array}{l}\text { Wilkins, Stephen; } \\
\text { Huisman, Jeroen }\end{array}$ & 2012 & 0 & 0 & 0 & 0 & 0 & 0 & 0 & 0 & 0 & 0 & 2 & 6 & 15 & 16 & 10 & 27 & 16 & 10 & 102 \\
\hline $\begin{array}{c}\text { Thuerer, Matthias; } \\
\text { Stevenson', Mark; Silva, } \\
\text { Cristovao; Land, Martin J.; } \\
\text { Fredendall, Lawrence D. }\end{array}$ & 2012 & 0 & 0 & 0 & 0 & 0 & 0 & 0 & 0 & 0 & 0 & 2 & 8 & 11 & 10 & 12 & 7 & 5 & 6 & 61 \\
\hline $\begin{array}{c}\text { Tian, Jing; Nakamori, } \\
\text { Yoshiteru; Wierzbicki, } \\
\text { Andrzej P. }\end{array}$ & 2009 & 0 & 0 & 0 & 0 & 0 & 0 & 0 & 1 & 3 & 6 & 3 & 5 & 7 & 10 & 10 & 6 & 10 & 4 & 65 \\
\hline Huisman, J; Currie, J & 2004 & 0 & 0 & 0 & 1 & 0 & 8 & 4 & 8 & 9 & 6 & 4 & 5 & 7 & 6 & 8 & 6 & 9 & 3 & 84 \\
\hline Knott, JH; Payne, AA & 2004 & 0 & 0 & 0 & 2 & 1 & 2 & 4 & 2 & 4 & 2 & 3 & 7 & 3 & 8 & 2 & 7 & 8 & 1 & 56 \\
\hline Ylijoki, OH; Mantyla, H & 2003 & 0 & 2 & 2 & 1 & 4 & 8 & 6 & 4 & 6 & 6 & 7 & 7 & 14 & 10 & 11 & 5 & 14 & 7 & 114 \\
\hline Lacy, FJ; Sheehan, BA & 1997 & 2 & 0 & 3 & 1 & 2 & 0 & 2 & 5 & 5 & 8 & 6 & 4 & 4 & 4 & 5 & 7 & 5 & 2 & 65 \\
\hline
\end{tabular}

Fonte: Elaborado pelos autores (2020). 
Dentre os autores mais citados da Web of Science, Tabela 1, registram-se as publicações nos periódicos: Time \& Society; Business Horizons; Higher Education; Research policy; Journal of knowledge management; Production and operations management; Journal of policy analysis and management.

Trata-se de expressiva informação para o fortalecimento e encaminhamento de novas pesquisas de âmbito de estudos, uma vez, que tais documentos de pesquisa, por meio de seus ambientes virtuais, possuem a capacidade de reunir discussões, pesquisadores e avaliadores, com o intuito de apresentar à comunidade acadêmica e à sociedade, estudos, modelos e efeitos práticos que transformem realidades e que ampliem as capacidades das organizações para satisfazer necessidades das pessoas e maximizar resultados.

Tal argumentação está alinhada ao apresentado por Bassi (2018), ao afirmar sobre pesquisas envolvendo a gestão universitária, esta autora discorre que são importantes estudos para o direcionamento de políticas educacionais que visem reduzir lacunas e fragilidades dos cursos e instituições de ensino, que busquem identificar maus desempenhos e melhorar sua atuação estratégica.

Objetivando evidenciar os temas mais discutidos entre o período de 1945 a 2020, sobre o termo "gestão universitária", compreendendo a base Web of Science, a Figura 1 expõe a rede de ocorrência de palavras organizadas em clusters, enfatizando as palavras-chave, a partir dos 492 artigos encontrados na base em comento, a qual se destacam 1.927 palavras, dispersas e embrenhadas em rede, em que foi possível fixar 173 encontros de termos, utilizando o software VOSviewer.

Figura 1. Rede de Ocorrência de Palavras (dados de 1945 - 2020) - Web of Science.

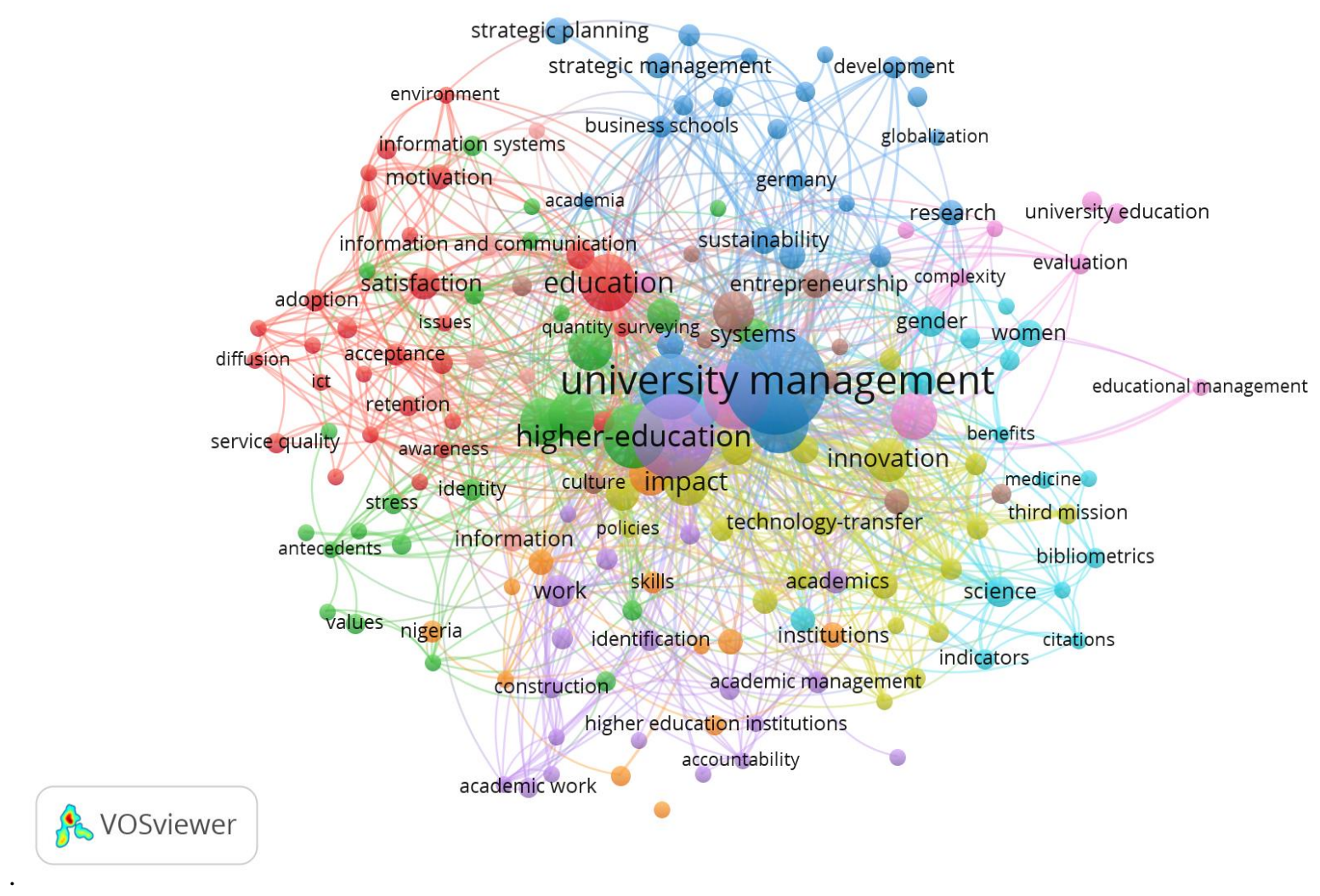

Fonte: Elaborado pelos autores com o VOSviewer, (2020).

A Figura 1 revela a conexão de palavras distribuídas em aglomerados, distribuídos em 9 clusters, destacando-se em cores mais quentes e intensas - conforme maior incidência dos termos - e cores mais frias e menos intensas - quando há menor 
incidência de termos - visualizando-se, neste trabalho, cores verde, vermelha, amarela, laranja, lilás, azul, rosa, azul turquesa e marrom. Na cor verde, destacam-se termos como identidade (identify), sistemas de informação (information systems), ensino superior (higer education), valores (values), estresse (stress) na cor vermelha, frisa-se motivação (motivation), satisfação (satisfaction), qualidade de serviço (service quality), consciência (awareness), na cor azul, os vocábulos planejamento estratégico e gestão estratégica (strategic planning e strategic management), e sustentabilidade (sustainability) são salientados; na cor amarela, termos como transferência de tecnologia (technology tranfer), terceira missão (third mission), e inovação (innovation); na cor laranja, instituições (institution); a cor roxa, ressalta expressões como identificação (identification), gestão acadêmica (academic management), prestação de contas (accountability).

Vale destacar, que diante da complexidade de estudos que envolvem a gestão universitária, observa-se que palavras centrais podem se correlacionar a diferentes abordagens de pesquisa, correlações, sistematizações e integrações de variáveis e conteúdos. Por esse motivo, torna-se relevante considerar tais expressões em estudos organizacionais sobre o contexto proposto, uma vez que a Figura 1, voltada à ocorrência de palavras sobre pesquisas cujo tema discorra sobre "gestão universitária”, evidencia importantes termos tratados em variadas pesquisas e tratos metodológicos pelo mundo.

Para isso, compreende-se que cada expressão ou algum termo exposto elencado, não se identifica como propriedade ou centralização de área do saber, especificamente, não cabendo interpretação de redução morfológica ao termo central ou ao objeto desta pesquisa. Tem-se, à vista disso, que sobre o objeto de pesquisa proposto e tema sobredito pesquisado, a listagem de termos e expressões sinaliza a importância dos conteúdos, abordagens e características envoltas nas discussões de gestão universitária para o período compreendido.

Tais análises e expressões remetem às idiossincrasias intrínsecas ao ambiente acadêmico, as quais abordam fatores relacionados a estas próprias organizações, seus valores, sistemas de informação, gestão acadêmica e prestação de contas, por exemplo. Também, são investigações no meio acadêmico que se voltam às perspectivas de comportamentos e às formas como seus usuários enxergam a atuação da instituição, diante de perspectivas e níveis de satisfação, estresse, motivação, consciência e identificação, registra-se, à guisa de aclaramento, que avaliações que perpassam instituições de ensino superior, abarcam de forma sistêmica e integrada no cerne de seu rendimento organizacional, tanto questões relacionadas à desenvoltura da instituição e seus regramentos, como também, atributos ligados à gestão de pessoas e sua atuação com os usuários.

Atinente aos sistemas de informação, Sabau et al. (2009) afirmam que estes não foram bem delineados e estruturados para a gestão universitária e seus desafios. Particularmente, no que reza sobre produção de relatórios, análises sistêmicas de dados históricos de alunos e apresentação de indicadores. Sobre essas questões, reside a importância de estudos diretos nessa área, sendo este, um dos termos mais utilizados em pesquisas, conforme Figura 1.

Necessita-se, portanto, de maior integração, a partir de desenvolvimento interno e integração, burilamentos direcionados à comunicação interna, à redução de processos manuais e maior utilização de processos eletrônicos, de modo que medidas e aperfeiçoamentos para tomada de decisão sejam facilitadas, promovendo maior assertividade e fortalecimento de atividades de planejamento e atendimento de servidores - professores e técnicos-administrativos - e discentes (Sabau et al., 2009).

Observa-se que há crescente enfoque de pesquisas orientadas à gestão do ensino superior, sobretudo, ao compromisso e potencial que as universidades podem ter no tocante à aspectos de inovação (Bayuo et al., 2020). Estas ações de desenvolvimento do meio universitário necessitam estar adequadas e em consonância com os próprios sistemas de inovação que por esses ambientes organizacionais podem ser gerados (Arocena et al., 2014).

Ainda, sobre caraterísticas e vocábulos detalhados em pesquisas da base Web of Science, acentua-se o termo Terceira Missão, a qual amplamente destacada e identificada como cunho comercial - de transferência de tecnologia - (Gibari et al. 
2018) expressões estas, introduzidas em uma concepção maior de "universidade empreendedora", contudo, o termo em comento não revela em seu âmago, o potencial inovativo e social destas organizações de ensino (Bayuo et al., 2020).

Argumentam, estes autores, que mesmo compondo a visão do empreendedorismo na terceira missão da universidade, estes espaços recebem manutenção financeira estatal, por meio do orçamento público para custeio acadêmico, constituindo-se este, como um fator de fortalecimento à identificação e sensibilidade do meio universitário à pauta social, seu incentivo e sua obrigação institucional (Bayuo et al., 2020).

No entanto, pormenorizando a expressão "universidades empreendedoras", Mainardes, Alves e Raposo (2011), afirmam que esse conceito de universidade, diz respeito a maior autonomia dessas organizações em comparação com o Estado, ao buscarem novas formas de financiamento e se ligarem mais à busca por soluções de problemas do mercado, e a comprometer-se mais com hodiernos problemas da sociedade, ampliando dessa forma, o engajamento da universidade com o desenvolvimento regional e local em que se insere.

Considerando a ocorrência de palavras expressas na Figura 2, agora, com classificação de termos por faixa de análise de 2013 a 2018 - período máximo disponível para geração dessa classificação - observa-se que ganham evidência termos como: universidade pública (public university), educação à distância (distance education), gestão ambiental (enviromental management), escolas de negócios (business schools), satisfação (satisfaction), equipe acadêmica (academic staff), motivação (motivation), estrutura (framework), (policy) e (policies), estas duas últimas, conforme Frey (2000), identificadas como relativas às ações e medidas finalizadas, a serem postas em prática.

Sobre essa análise da Figura 2, após envolver termos como responsabilidade social (social responsibility), entre 2015 a 2017, salienta-se expressões em destaque em publicações entre os anos de 2017 a 2018, como perspectiva (perspective), habilidades (skills), compromisso organizacional (organizational commitment), comprometimento (commitment), planejamento estratégico (strategic planing), sustentabilidade (sustainability), ambiente (environment), economia (economics). Observa-se que pesquisas voltam seus objetivos centrais de estudo a temas que também abrangem um contexto de responsabilidade social, além da busca por cumprimento de objetivos e planejamento, expressos na identificação de compromisso organizacional e no comprometimento.

Conforme Figura 2, no contexto de novas demandas sociais, sanitárias e de distintas exigências de formação, considera-se nesse bojo discursivo, a disponibilidade de variadas ferramentas, mídias e instrumentos de educação à distância. Sendo, essa temática, ponto de destaque em pesquisas desenvolvidas entre o período de 2013 a 2018. Para Muntean et al. (2011) a gestão universitária está voltada e dedicada à intensificação de estudos e avaliações do ensino à distância. Este fato pode ser visto como uma das razões de impulsão ao crescimento de pesquisas direcionadas às dinâmicas e contextura do ensino à distância. 
Figura 2. Ocorrência de Palavras - de 2013 a 2018 - Web of Science

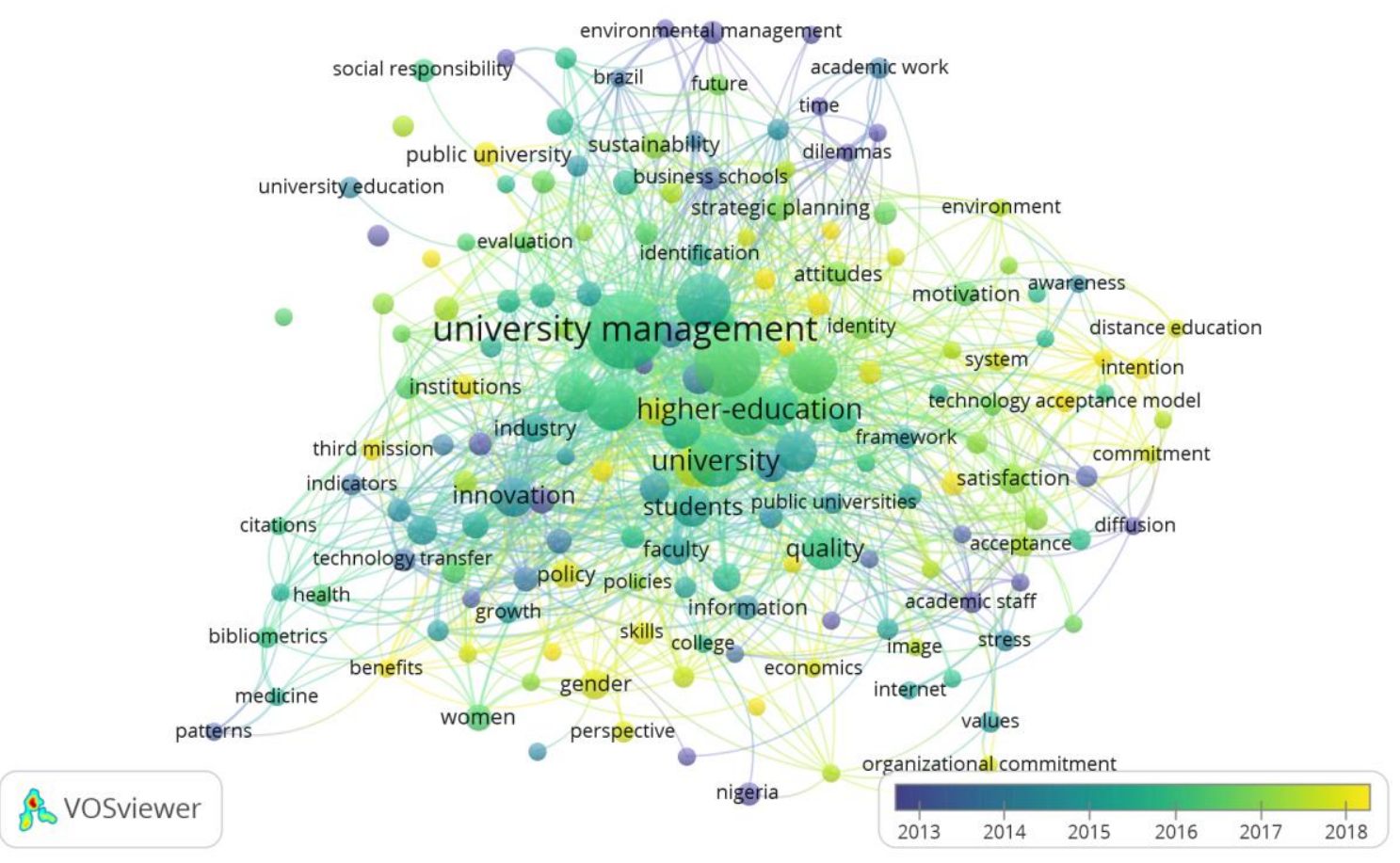

Fonte: Elaborado pelos autores com o VOSviewer, (2020).

Para Kaplan e Haenlein (2016), o ensino à distância dispõe de ferramentas vistas como primordial ao desenvolvimento de novas formas de remotas de instrução. Possibilitam diversas metodologias ativas, como salas de aulas invertidas, dispondo de tempo para discussões e disseminação de conteúdos de maneira remota. Tratam-se, portanto, de ferramentas que visam ampliar formas de disseminação da educação.

Essas argumentações são corroboradas diante de um cenário social, econômico e político fundamentado em novos desafios do mercado de trabalho, pautados por ampliação de propostas home office, propostas cada vez mais, diante de caóticas estruturas urbanas de trânsito nas grandes cidades (Rafalski \& Andrade, 2015), além da busca por maior inclusão educacional àqueles afastados dos centros com disponibilidade ao ensino superior - e enfoque na perseguição por qualidade de vida, redução de tempo e recursos para a realização de deslocamentos diários aos centros físicos educacionais.

Alusivo ao compromisso e desafios à gestão universitária, nesse bojo de critérios e características destacadas, os autores Pop-Vasileva, Baird e Blair (2014) inventariam que dentre seus substanciais responsabilidades encontra-se a necessidade de atração e retenção de pessoal com a qualidade às instituições. Sobre essas discussões, variadas perspectivas de pesquisas e de análises se constroem e se encadeiam.

A educação à distância passa a ser outro ponto de destaque nos artigos publicados na base Web of Science, relacionados à gestão universitária. Fato corroborado e evidenciado no Censo da Educação Superior do Brasil, elaborado pelo Ministério da Educação - MEC e Instituto Nacional de Estudos e Pesquisas Educacionais Anísio Teixeira - INEP, ao destacar considerando a faixa temporal de 2008 a 2018, que entre os anos de 2017 e 2018, houve acentuado crescimento no número de ingressos em cursos de graduação, tendo, sobretudo, para modalidade à distância, variação positiva de ingressos de $27,9 \%$ para o período avaliado, onde, para este mesmo biênio, sobre os cursos presenciais, a variação foi de -3,7\% (Brasil, 2019).

Reis e Cunha (2010) reforçam que apenas incluir alunos no ambiente educacional, não é o suficiente. É necessária a garantia de padrões mínimos de investimento, de modo que seja assegurada a garantia de acesso à educação com qualidade. 
Esta argumentação estende-se, em analogia à pauta da educação pública no Brasil, à necessidade de sofisticação e pesquisas que se voltem a ampliar garantias e a fornecer serviços públicos educacionais movidos pela perspectiva da eficiência e orientados pelos padrões de excelência na prestação de serviços ao cidadão.

Estas informações asseveram a importância de estudos e sua continuidade relativos à educação à distância no contexto da formação superior brasileira, conforme impulsionados por dados do Censo da Educação Superior no Brasil, enquanto que os ingressantes em cursos de graduação em 2008 centravam-se num percentual de 19,8\%, essa atuação aproximou-se de 40\%, correspondendo a 1.373 .321 ingressantes em cursos à distância em 2018, tendo 2.072 .614 como ingressantes em cursos presenciais para o mesmo ano.

\section{Considerações Finais}

Assinala-se que as percepções e estudos supramencionados desenvolvidos, não abordam de forma integrada as questões mencionadas no trabalho, a exemplo de estudos de inovação, satisfação e eficiência no ambiente público educacional. Tampouco, se centralizam sobre objetivos que abordem medidas de melhorias e eficiência no setor público educacional brasileiro por meio da modernização do setor público e melhor prestação de serviços.

No tocante ao problema de pesquisa registrado no trabalho, constata-se que as discussões sobre gestão universitária registradas na literatura, abordam, de forma individualizada e pontual, restritas discussões sobre satisfação e tecnologias no ensino público superior.

Sobre o objetivo geral apontado nessa pesquisa, nas análises, mediante registros de especificidades de artigos e autores mais citados - centrando citações entre 2000 a 2020 - ressalta-se que os estudos não dizem respeito a avaliações que se direcionem a fatores como eficiência, inovação e satisfação de usuários no contexto do ensino público superior federal brasileiro. Também, não apresenta estudos que integram e correlacionem essas citadas questões em suas pesquisas.

No que se refere aos objetivos específicos do trabalho, constata-se que as abordagens apresentadas, mediante consulta à base Web of Science, não expressam estudos orientados à temas como satisfação, inovação ou estudos sobre eficiência no contexto das universidades públicas brasileiras, fato este, o qual salienta a relevância de novas pesquisas e de maiores amadurecimentos em estudos na área.

Salientando, outras áreas relevantes para o desenvolvimento de análises que envolvam a gestão universitária, conforme expressões registradas no trabalho, os estudos publicados versam sobre variadas abordagens, como educação a distância, satisfação, motivação, qualidade dos serviços e planejamento estratégico.

Sobre a intenção da pesquisa e busca por sistematizar informações, sobre circunstâncias de artigos referentes à temática da gestão universitária, diante dos estudos analisados, observa-se que não há avaliação, sobre o cenário brasileiro, referente ao contexto da gestão universitária de modo que congregue estudos sobre fatores de inovação, eficiência e satisfação na educação superior.

O diagnóstico apresentado por meio desse estudo expõe a ausência de pesquisas e levantamentos que se encaminhem à análises concatenadas e sistemáticas sobre o cenário da gestão educacional e suas variáveis. Tal constatação salienta a necessidade de investigações que se foquem sobre a gestão universitária brasileira, fundamentadas em critérios como eficiência, inovação e satisfação dos usuários ante a prestação de serviços apresentados pelas organizações de ensino superior federais.

Como limitação desta pesquisa, aponta-se para um estudo fundamentado sobre a plataforma Web of Science, a qual mesmo diante de sua relevância em pesquisas em correlatas e interdisciplinares áreas do conhecimento, podem não representar o cenário global de artigos e perspectivas de assuntos sobre a gestão universitária e fatores de influência. 
Como sugestões para trabalhos futuros registra-se a realização de análise na plataforma Web of Science sendo replicada em outra plataforma de pesquisa, como a Scopus. De modo a ampliar a abordagem avaliativa e obter visões cada vez mais completas sobre o que tem sido discutido na literatura a respeito da gestão universitária. Além de propor novos recortes temporais de análise sobre pesquisas com a temática da gestão universitária, por exemplo, mediante avaliação de 1980 a 2022 em ambas as plataformas de pesquisa citadas.

Ainda, como contribuição da pesquisa, assinala-se que, mesmo diante de diversificadas propostas, as pesquisas disponíveis na Web of Science assumem a importância de subsidiar e fortalecer percepções, argumentações e investigações, as quais incitam novos estudos e influenciam a construção de métodos e diagnósticos sobre o contexto universitário.

\section{Referências}

Arocena, R., Göransson, B. \& Sutz, J. (2014). Knowledge policies and universities in developing countries: Inclusive development and the "developmental university". Technology in Society, 1-11. http://dx.doi.org/10.1016/j.techsoc.2014.10.004

Bassi, F. (2018). Dynamic clustering to evaluate satisfaction with teaching at university. International Journal of Educational Management, 32(6), 1070-1081. https://doi.org/10.1108/IJEM-07-2017-0162

Bayuo, B. B., Chaminade, C., \& Göransson, B. (2020). Unpacking the role of universities in the emergence, development and impact of social innovations - A systematic review of the literature. Technological Forecasting \& Social Change, 155(1), 1-3. Disponível em: https://doi.org/10.1016/j.techfore.2020.120030

Brasil. Decreto n ${ }^{\circ}$ 5.378, de 23 de fevereiro de 2005. http://www.planalto.gov.br/ccivil_03/_Ato2004-2006/2005/Decreto/D5378.htm

Brasil. Decreto no 9.094, de 17 de julho de 2017. http://www.planalto.gov.br/ccivil_03/_Ato2015-2018/2017/Decreto/D9094.htm

Brasil. Instituto Nacional de Estudos e Pesquisas Educacionais Anísio Teixeira (INEP). https://download.inep.gov.br/educacao_superior/censo_su perior/documentos/2019/censo_da_educacao_superior_2018-notas_estatisticas.pdf

Brasil. Lei n ${ }^{\circ}$ 9.394, de 20 de dezembro de 1996. http://www.planalto.gov.br/Ccivil_03/leis/L9394.htm

Brasil. Lei no 13.168, de 6 de outubro de 2015. http://www.planalto.gov.br/ccivil_03/_Ato2015-2018/2015/Lei/L13168.htm

Brasil. Lei no 13.460, de 26 de junho de 2017. http://www.planalto.gov.br/ccivil_03/_ato2015-2018/2017/Lei/L13460.htm

Csomós, G. (2020). Introducing recalibrated academic performance indicators in the evaluation of individuals' research performance: A case study from Eastern Europe. Journal of Informetrics, 14(4), 1-38. https://doi.org/10.1016/j.joi.2020.101073

Frey, K. (2000). Políticas públicas: um debate conceitual e reflexões referentes à prática da análise de políticas públicas no Brasil. Planejamento e Políticas Públicas, 21(1), 211 - 260. http://www.ipea.gov.br/ppp/index.php/PPP/article/viewFile/89/158

Huisman, J. \& Currie, J. (2004). Accountability in higher education: Bridge over troubled water?. Higher Education, 48(1), 529-551, 2004. https://doi.org/10.1023/B:HIGH.0000046725.16936.4c

Kaplan, A. M. \& Haenlein, M. (2016). Higher education and the digital revolution: About MOOCs, SPOCs, social media, and the Cookie Monster. Business Horizons, 59(4), 441-450. https://doi.org/10.1016/j.bushor.2016.03.008

Knott, J. H. \& Payne, A. A. (2004). The impact of state governance structures on managemente and performance of public organizations: a study of higher education institutions. Jornal of policy Analysis and Management, 23(1), 13-30. http://www.jstor.org/stable/3326188

Lacy, F. J. \& Sheehan, B. A. (1997). Job satisfaction among academic staff: An international perspective. Higher Education, 34(1), 305-322. https://doi.org/10.1023/A:1003019822147

Li, Y., Gu, Y. \& Liu, C. (2018). Prioritising performance indicators for sustainable construction and development of university campuses using an integrated assessment approach. Journal of Cleaner Production, 202(1), 959-968. https://doi.org/10.1016/j.jclepro.2018.08.217

Mainardes, E. W., Alves, H. \& Raposo, M. (2011). The process of change in university management: from the "ivory tower" to entrepreneurialism. Transylvanian Review of Administrative Sciences, 33(1), 124-149. https://www.rtsa.ro/tras/index.php/tras/article/view/287

Muntean, M., Bologa, A., Bologa, R. \& Florea, A. (2011). The use of multidimensional models to increase the efficiency of management support systems. International Journal of Mathematical Models and Methods in Applied Sciences, 5(8), 1-11. https://www.naun.org/multimedia/NAUN/ijmmas/17-207.pdf

O'kane, C., Mangematin, V., Geoghegan, W. \& Fitzgerald, C. (2015). University technology transfer offices: The search for identity to build legitimacy. Research Policy, 44(2), 421-437. http://dx.doi.org/10.1016/j.respol.2014.08.003

Oliveira, E. F. T. de. (2018). Estudos métricos da informação no Brasil: indicadores de produção, colaboração, impacto e visibilidade. Cultura Acadêmica. 184 p. https://www.marilia.unesp.br/Home/Publicacoes/estudos-metricos-da-informacao-no-brasil---e-book.pdf 
Research, Society and Development, v. 11, n. 2, e36911226023, 2022

(CC BY 4.0) | ISSN 2525-3409 | DOI: http://dx.doi.org/10.33448/rsd-v11i2.26023

Pereira, R. S., Santos, I. C., Oliveira, K. D. S. \& Leão, N. C. A. (2019). Metanálise como instrumento de pesquisa: Uma revisão sistemática dos estudos bibliométricos em Administração. Revista de Administração Mackenzie, 20(5), 2019. http://www.scielo.br/scielo.php?script=sci_arttext\&pid=S1678$69712019000500301 \& \operatorname{lng}=\mathrm{en} \& \mathrm{nrm}=$ iso

Pop-vasileva, A., Baird, K. \& Blair, B. (2014). The Work-related Attitudes of Australian Accounting Academics. Accounting Education: An International Journal, 23(1), 1-21. http://dx.doi.org/10.1080/09639284.2013.824689

Prodanov, C. C. \& Fretas, E. C. (2013). Metodologia do trabalho científico: métodos e técnicas da pesquisa e do trabalho acadêmico. (2a ed.), Feevale.

Rafalski, J. C. \& Andrade, A. L. (2015). Home-Office: Aspectos Exploratórios do Trabalho a partir de Casa. Trends in Psychology, 23 (2), 431-441. http://dx.doi.org/10.9788/TP2015.2-14

Reis, F. P. G. dos. \& Cunha, D. O. (2010). Políticas públicas \& desenvolvimento regional. Campina Grande: EDUEPB.

Sabau, G., Munten, M., Bologa, A., Bologa, R. \& Surcel, T. (2009). An Evaluation Framework for Higher Education ERP Systems. Wseas Transactions on Computers, 8(11), 1-10. http://www.wseas.us/e-library/transactions/computers/2009/29-795.pdf

Soares, J. R., Bordin, R. \& Rosa, R. S. (2019). Indicadores de gestão e de qualidade nas instituições federais de ensino superior brasileiras - $2009-2016$. Revista Eletrônica de Administração, 25(2). http://dx.doi.org/10.1590/1413-2311.250.95100

Ylijoki, O., \& Hans, M. (2003). Conflicting Time Perspectives in Academic Work. Time \& Society, 12(1), 55-78. https://doi.org/10.1177/0961463X03012001364 